\title{
Avaliação da incapacidade e saúde de pacientes com doença renal crônica submetidos a hemodiálise
}

\section{Evaluation of disability and health of patients with chronic renal disease undergoing hemodialysis}

\author{
Thatyane Cruvinel Silva Hungria ${ }^{1}$, Gustavo Silva de Azevedo ${ }^{2}$, \\ Amanda Rafaella Araújo de Campos ${ }^{3}$, Amélia Cristina Stival Duarte ${ }^{4}$
}

http://dx.doi.org/10.11606/issn.2238-6149.v31i1-3p31-37

\begin{abstract}
Hungria TCS, Azevedo GSA, Campos ARA, Duarte ACS. Avaliação da incapacidade e saúde de pacientes com doença renal crônica submetidos a hemodiálise. Rev Ter Ocup Univ São Paulo. 2020 jan.-dez.;31(1-3):31-7.
\end{abstract}

RESUMO: Introdução: A doença renal crônica(DRC) corresponde à redução irreversível da função renal, provoca um grande impacto para a pessoa acometida e suas funcionalidades básicas ficam prejudicadas, resultando em mudanças importantes no seu estilo de vida ${ }^{1}$. Objetivo: Avaliar o grau de incapacidade e saúde de pacientes com doença renal crônica submetidos à hemodiálise. Metodologia: Trata-se de um estudo de campo, do tipo transversal, com amostra de conveniência, para a avaliação sociodemográfica, clínico funcionais e de desempenho funcional dos pacientes DRC correlacionados ao questionário Word Health Organization Disability Assessment Schedule - WHODAS versão 2.0. Resultados: Os participantes do estudo $(n=59)$, com predominância de homens $(67,79 \%)$, e média de idade de 51,75 \pm 00 anos, apresentaram comorbidades associadas à DRC, tendo destaque para hipertensão arterial sistêmica $(67,79 \%)$ e diabetes mellitus (33,89\%). A mobilidade dos pacientes mensuradas pelo Time Up and Go - TUG teste possuiu correlação com o WHODAS nos domínios de: cognição, mobilidade, autocuidado e participação. Houve correlação inversamente proporcional entre a força de preensão palmar e os domínios de mobilidade, autocuidado e atividades domésticas. O grau de dor verificado pela escala EVA teve representatividade junto aos domínios de cognição, mobilidade, autocuidado, relações interpessoais, participação e atividades domésticas. Conclusão: Verificase os amplos prejuízos na funcionalidade de pacientes hemodialíticos; e a Fisioterapia, por dispor de vários recursos de avaliação e intervenção, poderá amenizar a incapacidade e favorecer a saúde dessa população.

DESCRITORES: Doença renal crônica; Hemodiálise; Desempenho físico funcional; Fisioterapia.
Hungria TCS, Azevedo GSA, Campos ARA, Duarte ACS. Evaluation of disability and health of patients with chronic renal disease undergoing hemodialysis. Rev Ter Ocup Univ São Paulo. 2020; Jan-Dec.;31(1-3):31-7.

ABSTRACT: Introduction: Chronic kidney disease (CKD) corresponds to an irreversible reduction in kidney function, causes a great impact to the person affected and its basic functionalities are impaired, resulting in important changes in their lifestyle1. Objective: To assess the degree of disability and health of patients with chronic kidney disease undergoing hemodialysis. Methodology: This is a cross-sectional field study, with a convenience sample, for the sociodemographic, clinical and functional performance assessment of CKD patients correlated to the Word Health Organization Disability Assessment Schedule - WHODAS version 2.0 questionnaire. Results: Study participants $(n=59)$, with a predominance of men $(67.79 \%)$, and a mean age of $51.75 \pm 00$ years, presented comorbidities associated with $\mathrm{CKD}$, with emphasis on systemic arterial hypertension $(67,79 \%)$ and diabetes mellitus (33.89\%). Patient mobility measured by the Time Up and Go - TUG test was correlated with WHODAS in the following domains: cognition, mobility, self-care and participation. There was an inversely proportional correlation between handgrip strength and the domains of mobility, self-care and domestic activities. The degree of pain verified by the EVA scale was representative of the domains of cognition, mobility, self-care, interpersonal relationships, participation and domestic activities. Conclusion: There are extensive losses in the functionality of hemodialysis patients; and Physiotherapy, by having several assessment and intervention resources, can alleviate the disability and favor the health of this population.

KEYWORDS: Chronic kidney disease; Hemodialysis; Functional physical performance; Physiotherapy.

Trabalho de Conclusão de Residência apresentado à Secretaria de Estado da Saúde de Goiás e ao Centro Universitário de Anápolis UniEVANGÉLICA para obtenção do título de Especialista em Endocrinologia.

1. Fisioterapeuta no Programa de Residência em Area Profissional da Saúde - Atenção Clínica Especializada em Endocrinologia. Orcid: 0000-0001-5005-1111. Email: thaatyc@gmail.com

2. Fisioterapeuta no Hospital Estadual Geral de Goiânia Dr. Alberto Rassi. Orcid: 0000-0003-1788-3586. Email: gusaz@gmail.com

3. Fisioterapeuta no Programa de Residência em Area Profissional da Saúde - Atenção Clínica Especializada em Endocrinologia. Orcid: 0000-0003-1744-3492. Email: amandarafaella12@gmail.com

4. Nutricionista no Hospital Estadual Geral de Goiânia Dr. Alberto Rassi. Orcid: .0000-0003-0878-2441. Email: ameliastival@hotmail.com. Endereço para correspondência: Thatyane C S Hungria. Rua 8, Edifício Veneza 2, Setor Oeste, Goiânia- Goiás. 


\section{INTRODUÇÃO}

Amanifestação patológica lenta e progressiva, com etiologias distintas; apresenta redução irreversível da função renal prejudicando as funcionalidades básicas além de provocar um acúmulo de resíduos de degradação metabólica circulantes no sangue ${ }^{1}$.Portanto, altera-se nesse órgão a eliminação de toxinas derivadas do metabolismo, despertando o prejuízo na regulação do balanço hidroeletrolítico, do $\mathrm{pH}$ sanguíneo e interferindo na homeostase corporal ${ }^{2}$.

A diretriz da fundação Kidney Disease Outcome Quality Initiative (KDOQI) define o paciente com DRC o indivíduo em que se observa a Taxa de Filtrado Glomerular (TFG) menor que $60 \mathrm{~mL} / \mathrm{min} / 1,73 \mathrm{~m}^{2}$. Também se considera portador de DRC resultados acima desse valor, associado a pelo menos um marcador de comprometimento renal, tais como: alteração nos níveis de ureia e/ou creatinina por um tempo prolongado, superior de três meses ${ }^{3}$.

A Sociedade Brasileira de Nefrologia observou no período 2005-2015 aumento significativo do número de pacientes em procedimento dialítico ${ }^{4}$. Desta forma, quando a lesão renal atinge graus mais avançados, determinados como estágio V, são necessárias intervenções invasivas para reproduzir as funções do rim prejudicado, uma vez que apenas $15 \%$ da funcionalidade do órgão cumpre sua função nessa fase 5 .

Para suprir parcialmente a função renal debilitada, alguns procedimentos terapêuticos utilizados no tratamento da DRC são essenciais para a manutenção da vida. Dentre as terapias renais substitutivas, encontram-se o transplante renal, e a diálise; sendo essa dividida em hemodiálise e diálise peritoneal ${ }^{6}$. No entanto, essas abordagens diminuem os sintomas presentes, porém não revertem a doença.

Essa repercussão no sistema renal provoca um grande impacto para a pessoa, resultando em mudanças importantes no seu estilo de vida, a nível corporal, espiritual e mental, ocasionando perda da autonomia e capacidade de locomoção, estresse e ansiedade, afastamento do convívio social, limitações no trabalho, sentimento de risco de vida iminente, restrições na ingestão oral de líquidos e alimentos, diminuição da libido, dentre vários fatores limitantes ${ }^{5,6}$.

A Organização Mundial de Saúde (OMS) vem desenvolvendo modelos classificadores dos fenômenos envolvidos na funcionalidade, incapacidade, saúde e deficiência, tais como a Classificação Internacional de Funcionalidade, Incapacidade e Saúde (CIF, 2001) e o World Health Disability Assessment Schedule (WHODAS versão 2.0), o qual é uma versão reduzida e prática, que viabiliza sua aplicabilidade e facilita sua inserção nos serviços de saúde ${ }^{7}$.
Dessa forma, pretende-se identificar quais as variáveis envolvidas nas limitações dos pacientes dialíticos, que os impedem de viver em desempenho pleno.

\section{OBJETIVOS}

Avaliar o grau de incapacidade e saúde de pacientes com doença renal crônica submetidos à hemodiálise e correlacionar com variáveis clínico-funcionais.

\section{METODOLOGIA}

Trata-se de um estudo de campo, do tipo transversal, a amostra é de conveniência, composta de 59 pacientes portadores de doença renal crônica que estiveram submetidos a hemodiálise no Hospital Estadual Dr. Alberto Rassi-HGG, sendo internados em enfermaria na ala de hemodiálise ou em acompanhamento ambulatorial. Foram incluídos no estudo pacientes de ambos sexos, diagnosticados com insuficiência renal crônica ou doença renal crônica avançada (grau 5), que realizassem hemodiálise, pré transplante ou pós transplantados com perda de enxerto no Hospital Geral de Goiânia - HGG, que possuam quadro cognitivo preservado, acima de 18 anos de idade e que aceitarem participar voluntariamente do estudo assinando o Termo de Consentimento Livre e Esclarecido (TCLE). Pacientes com dificuldade de compreensão, em uso de medicação psicotrópica (benzodiazepínicos), ou classificados em cuidados paliativos foram excluídos da amostra.

A entrevista e aplicação dos testes foi realizada por um único entrevistador, que identificava os indivíduos elegíveis para a pesquisa através de busca em prontuário. Antes da sessão de hemodiálise, foi iniciada a aplicação dos testes funcionais de mobilidade Timed up and Go (TUG) e o Teste de força de preensão palmar (FPP). Para mensurar a força muscular dos participantes foi realizado o teste de FPP, através do dinamômetro do tipo pneumático, do modelo Saehan Grip $^{\circledR}$, a aferição de força foi padronizada utilizando-se sempre a mão dominante do paciente.

A altura em metros (m) e peso corporal (peso seco em $\mathrm{kg}$ ) foram coletados a partir de dados anexados em prontuário, atualizados a cada sessão de hemodiálise. Para a classificação do IMC, foi considerado os valores recomendados pela OMS, diferindo entre a população com mais de 60 anos.

Logo em seguida, foi realizada a aplicação dos questionários em forma de entrevista estruturada no decorrer da sessão de hemodiálise; sendo a primeira parte da entrevista uma ficha de avaliação, onde se avalia dados sociodemográficos e clínico funcionais.

Por fim, o nível de funcionalidade e grau de incapacidade foram obtidos através do instrumento World 
Health Organization Disability Assessment Schedule (WHODAS 2.0), versão 36 itens. O questionário avalia a incapacidade para realizar atividades da vida diária nos últimos 30 dias, e é composto por seis domínios: 1) "Cognição, 2) "Mobilidade", 3)"Autocuidado", 4) "Relacionamento Interpessoal", 5) "Atividades de Vida" (atividades doméstica, escolares e trabalho), e 6) "Participação".

\section{Análise Estatística}

A análise estatística foi realizada no programa IBM SPSS Statistics for Windows 16.0. A normalidade das variáveis foi testada por meio do teste Shapiro-Wilk. A distribuição das médias entre os sexos foi feita por meio do teste de Mann-Whitney; e correlação entre as variáveis e os domínios do WHODAS 2.0 foi analisada por meio do coeficiente de correlação de Spearman. Para todos os testes, considerou-se um nível de significância de $5 \%$ (valor $p<0,05$ ) e intervalo de confiança de $95 \%$.

\section{RESULTADOS}

A amostra foi composta por 59 pacientes, sendo representada em $67,79 \%(n=40)$ pelo sexo masculino. A média de idade foi de $51,75 \pm 0,0$ anos. A média do IMC foi de $(25,49 \pm 0,48)$, classificado como sobrepeso na população com menos de 60 anos, e eutrófico na população idosa.

Em relação as comorbidades associadas, 37,28\% (22) tem no mínimo 3 doenças diagnosticadas, sendo $33,89 \%(n=20)$ portador de Diabetes Mellitus, enquanto a comorbidade mais frequente foi a Hipertensão Arterial, com $67,79 \%(\mathrm{n}=40)$.
A quantidade de quedas sofridas nos últimos 12 meses foi baixa, visto que $81,35 \%(n=48)$ dos entrevistados revelaram que não sofreram nenhuma queda nesse período. Os resultados do TUG teste revelaram um baixo risco para queda com 96,07\% ( $\mathrm{n}=49)$ dos pacientes conseguindo finalizar o teste em menos de 20 segundos.

Quanto ao local e índice de dor, 40,67\% dos participantes relataram sentir dores em membros inferiores e, considerando a escala EVA de zero a dez. Na avaliação da força de preensão palmar, os valores para o sexo masculino $(30,48 \pm 5,19)$, obteve uma média superior quando comparado com o sexo feminino $(13,69 \pm 4,0)$.

O tempo de o diagnóstico de DRC apresenta uma

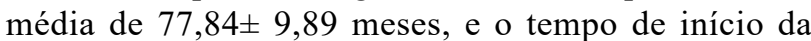
hemodiálise teve uma média de 34,49 meses. No geral, os valores de exames clínicos correspondem à hemoglobina em $8,83 \pm 2,33 \mathrm{~g} / \mathrm{dL}$ que se encontra abaixo do valor de referência para adultos, entretanto $88,13 \%(\mathrm{n}=52)$ dos pacientes submetidos a hemodiálise apresentaram valores $\leq 11 \mathrm{~g} / \mathrm{dL}$. As médias de ureia $(107,2 \pm 0,42) \mathrm{mg} / \mathrm{dL}$ e creatinina $(6,25 \pm 1,35) \mathrm{mg} / \mathrm{dL}$ se encontraram acima dos valores considerados dentro da normalidade.

Verificou-se o grau de incapacidade dos pacientes submetidos a hemodiálise, a partir do questionário WHODAS 2.0, foi considerado o valor total de cada domínio, que apresentou dificuldade leve para os domínios de cognição, relações interpessoais e atividades no trabalho.

Apontuação total do WHODAS demonstrou dificuldade moderada nos participantes dessa amostra (Tabela 1) e verificada a distribuição das médias entre os sexos (Tabela 2), com diferença significativa na força de preensão palmar e níveis de ureia.

Tabela 1 - Classificação WHODAS 2.0 e respectivos escores de incapacidade dos pacientes submetidos a hemodiálise (n=59), Goiânia, 2020.

\begin{tabular}{lccc}
\hline Domínios & Média \pm DP & Incapacidade \% & Escore \\
\hline Cognição & $4,14 \pm 3,75$ & 20,67 & DL \\
Mobilidade & $5,92 \pm 5,43$ & 36,97 & DM \\
Autocuidado & $2,59 \pm 2,88$ & 25,93 & DM \\
Relações interpessoais & $2,12 \pm 2,14$ & 17,66 & $\mathrm{DL}$ \\
Atividades domésticas & $5,56 \pm 4,15$ & 55,59 & $\mathrm{DG}$ \\
Atividades do trabalho & $1,14 \pm 3,44$ & 8,11 & $\mathrm{DL}$ \\
Participação & $10,27 \pm 5,27$ & 42,80 & $\mathrm{DM}$ \\
WHODAS 2.0 TOTAL & $32,36 \pm 25,68^{\mathrm{a}}$ & $30,32^{\mathrm{a}}$ & $\mathrm{DM}$ \\
& $31,58 \pm 17,85$ & $23,32^{\mathrm{b}}$ & $\mathrm{DM}^{\mathrm{b}}$ \\
\hline
\end{tabular}

WHODAS 2.0: World Health Organization Disability Assessment Schedule 2.0. a Total considerando participantes que trabalham, $\mathrm{n}=11$, sendo $21 \%$ da amostra ${ }^{b}$. Total considerando participantes que não trabalham. Incapacidade (\%) expressa o percentual de perda em cada domínio. Score: Score de incapacidade no WHODAS2.0: D.G. (dificuldade grave); D.M. (dificuldade moderada); D.L. (dificuldade leve). 
Hungria TCS, et al. Avaliação da incapacidade e saúde de pacientes com doença renal crônica. Rev Ter Ocup Univ São Paulo. 2020 jan.-dez.;31(1-3):31-7.

Tabela 2 - Nível de evidência para correlação entre os domínios do WHODAS 2.0 com o perfil clínico-funcional pacientes submetidos a hemodiálise (n=59), Goiânia, 2020.

\begin{tabular}{|c|c|c|c|c|c|}
\hline \multirow{3}{*}{$\begin{array}{l}\text { Variáveis } \\
\text { Índice Massa Corporal }\end{array}$} & \multicolumn{4}{|c|}{ Média \pm desvio padrão } & \multirow{3}{*}{$\begin{array}{r}\text { Valor } \mathbf{p}^{*} \\
0,168\end{array}$} \\
\hline & \multicolumn{2}{|c|}{ Masculino } & \multicolumn{2}{|c|}{ Feminino } & \\
\hline & 24,99 & $\pm 7,27$ & 26,53 & $\pm 5,92$ & \\
\hline Força Preensão Palmar & 30,48 & $\pm 05,19$ & 13,69 & $\pm 4,00$ & $<0,001$ \\
\hline Hemoglobina & 8,85 & $\pm 1,73$ & 8,78 & $\pm 1,87$ & 0,927 \\
\hline Ureia & 115,57 & $\pm 58,21$ & 89,63 & $\pm 37,17$ & $\mathbf{0 , 0 3 9}$ \\
\hline Creatinina & 6,84 & $\pm 3,83$ & 4,98 & $\pm 2,59$ & 0,078 \\
\hline Escala visual analógica de dor & 2,65 & $\pm 3,48$ & 3,32 & $\pm 3,74$ & 0,497 \\
\hline Time Up and Go Teste & 11,49 & $\pm 4,83$ & 12,56 & $\pm 3,05$ & 0,082 \\
\hline \multicolumn{6}{|c|}{ Domínios WHODAS } \\
\hline Cognição & 3,73 & $\pm 3,46$ & 5,00 & $\pm 4,26$ & 0,319 \\
\hline Mobilidade & 6,03 & $\pm 5,64$ & 5,68 & $\pm 5,11$ & 0,922 \\
\hline Autocuidado & 2,63 & $\pm 2,80$ & 2,53 & $\pm 3,12$ & 0,887 \\
\hline Relações interpessoais & 1,95 & $\pm 2,07$ & 2,47 & $\pm 2,29$ & 0,394 \\
\hline Atividades Domésticas & 5,58 & $\pm 4,19$ & 5,53 & $\pm 4,18$ & 0,907 \\
\hline Atividades do Trabalho & 1,20 & $\pm 3,52$ & 1,00 & $\pm 3,35$ & 0,840 \\
\hline Participação & 10,40 & $\pm 5,53$ & 10,00 & $\pm 4,81$ & 0,776 \\
\hline Total WHODAS & 31,50 & $\pm 20,50$ & 32,21 & $\pm 16,97$ & 0,839 \\
\hline
\end{tabular}

*Teste de Mann-Whitney

As relações interpessoais do WHODAS têm correlação com idade, IMC e EVA dos participantes desse estudo; já o TUG teste se correlaciona respectivamente a itens do WHODAS em especial à mobilidade, cognição, autocuidado e participação. Ainda sobre as avaliações físicas, a FPP tem relação quanto ao autocuidado e também à mobilidade desse grupo.
O nível de dor mensurado pela escala EVA corroborou em grande parte dos domínios avaliados, com exceção do item "atividades de trabalho". Já os exames laboratoriais analisados, apenas a creatinina se correspondeu à mobilidade e autocuidado (Tabela 3).

Tabela 3 - Correlação do perfil clínico-funcional e incapacidade dos pacientes DRC submetidos a hemodiálise (n=59), Goiânia,2020.

\begin{tabular}{lcccccccc}
\hline \multirow{2}{*}{ Variáveis } & \multicolumn{7}{c}{ Domínios do WHODAS 2.0 } \\
\cline { 2 - 9 } & Cognição & Mobilidade & Autocuidado & $\begin{array}{c}\text { Relações } \\
\text { interpessoais }\end{array}$ & $\begin{array}{c}\text { Atividades } \\
\text { Domésticas }\end{array}$ & $\begin{array}{c}\text { Atividade } \\
\text { Trabalho }\end{array}$ & Participação & Total \\
\hline Idade & 0,190 & 0,207 & 0,197 & $\mathbf{0 , 2 6 4 *}$ & $\mathbf{0 , 2 5 9 *}$ & $-0,217$ & 0,059 & 0,173 \\
IMC & $-0,082$ & 0,123 & 0,133 & $\mathbf{0 , 2 6 0 *}$ & 0,073 & $-0,014$ & $-0,101$ & 0,02 \\
TUG & $\mathbf{0 , 3 4 9 *}$ & $\mathbf{0 , 4 5 0 *}$ & $\mathbf{0 , 4 5 9 *}$ & $0,367 *$ & 0,270 & $-0,148$ & $\mathbf{0 , 2 9 1 *}$ & $\mathbf{0 , 4 1 2 *}$ \\
FPP & $-0,137$ & $\mathbf{- 0 , 3 2 1 *}$ & $\mathbf{- 0 , 3 2 1 *}$ & $-0,192$ & $\mathbf{- 0 , 3 0 9 *}$ & 0,145 & $-0,185^{*}$ & $\mathbf{- 0 , 3 0 3 *}$ \\
EVA & $\mathbf{0 , 3 4 5 *}$ & $\mathbf{0 , 3 0 8 *}$ & $\mathbf{0 , 3 4 9 *}$ & $\mathbf{0 , 3 8 6 *}$ & $\mathbf{0 , 2 5 8 *}$ & 0,127 & $\mathbf{0 , 2 6 4} *$ & $\mathbf{0 , 3 9 9 *}$ \\
Hb & $-0,045$ & $-0,019$ & $-0,128$ & 0,031 & 0,073 & $-0,062$ & $-0,064$ & $-0,045$ \\
Ureia & $-0,142$ & $-0,169$ & $-0,131$ & $-0,083$ & $-0,137$ & 0,129 & $-0,08$ & $-0,143$ \\
Creatinina & $-0,235$ & $\mathbf{- 0 , 3 2 6 *}$ & $\mathbf{- 0 , 3 0 2 *}$ & $-0,151$ & $-0,148$ & 0,089 & $-0,218$ & $-\mathbf{0 , 2 9 9 *}$ \\
\hline
\end{tabular}

* $\mathrm{p}<0,05$ (teste de correlação de Spearman) IMC: Índice de Massa Corporal $\left(\mathrm{kg} / \mathrm{m}^{2}\right)$; TUG: Teste Timed Up and Go; Força de Preensão Palmar: kgf; EVA: Escala visual analógica de dor; Hb: hemoglobina: (g/dL); Ureia e Creatinina (mg/dL). 


\section{DISCUSSÃO}

Observa-se na maioria dos estudos realizados com pacientes DRC, verifica sua associação com a idade avançada e geralmente em pacientes idosos ${ }^{7}$; o que não corrobora com os resultados do presente estudo, visto que a média de idade foi menor que 60 anos.

Os participantes tiveram predomínio do sexo masculino, assim como no estudo realizado em 2018 por Castro et al. ${ }^{7}$ com a mesma população e instrumento de avaliação de incapacidade; o tempo de diagnóstico de DRC foi aproximado entre estudos, sendo de 77,84 meses e 71,05 meses respectivamente.

Porém o tempo de tratamento do atual estudo se mostrou inferior se comparado com levantamento realizado pelo mesmo estudo, isso pode indicar um grupo com melhor manejo da doença principalmente em relação a prevenção da progressão da doença, tendo visto um desfecho mais tardio do início da hemodiálise.

A Hipertensão Arterial Sistêmica, considerada uma afecção totalmente relacionada a DRC, se faz presente em $67,79 \%$ dessa amostra; ademais a HAS pode ser tanto a causa quanto uma consequência da doença renal ${ }^{7}$. A doença renal muitas vezes é o desfecho de outras comorbidades, como o diabetes mellitus, devido suas complicações macro e microvasculares. Nessa amostra observamos $33,89 \%(\mathrm{n}=20)$ de pacientes diabéticos, sendo considerada valores acima de $20 \%$ considerada uma alta prevalência de Doença Renal diabética (DRD) 9 .

Quanto aos valores laboratoriais de creatinina e uréia, identificamos esses marcadores renais bastante elevados nesse grupo. Essa alteração em conjunto indica um estado patológico renal, considerada uma afecção totalmente relacionada a $\mathrm{DRC}^{10}$.

O baixo valor sérico de hemoglobina dos participantes desse estudo está concomitante com a literatura, e indica uma situação de anemia frequente no paciente em hemodiálise; e muitas vezes essa afecção surge precocemente no curso da doença com tendência a aumentar à medida que ocorre diminuição da função renal; isso acontece devido ao quadro de inflamação que inibe a absorção duodenal de ferro ${ }^{11}$.

Além disso, grande parte dos participantes nesse estudo mostraram-se com perfil sedentário em relação à atividade física. Isso pode ser elucidado por apresentarem alterações da capacidade física como a sarcopenia, fadiga, câimbras, prostração e outros empecilhos, decorrentes da doença e também da hemodiálise ${ }^{12}$.

Considera-se a força global com valores preditores de $\geq 37 \mathrm{~kg}$ para homens $\mathrm{e} \geq 21 \mathrm{Kg}$ para mulheres ${ }^{13}$, a amostra teve média de $30,48 \pm 5,19 \mathrm{~kg}$ para homens e $13,69 \pm 4,0 \mathrm{~kg}$ de força para mulheres, o que proporcionalmente sugere uma grande perda de força presente em ambos os sexos. Este fato pode ser demonstrado na tabela 2, onde apresenta os níveis elevados de uréia em ambos os sexos $(p<0,039)$, o que pode estar relacionado a redução da força de preensão devido deteriorazação de fibras musculares ${ }^{13,14}$.

Bem como encontrado na literatura, o IMC dos pacientes estudados equiparou-se a classificação de eutrofia estabelecidos pela OMS, apesar de frequentemente esses pacientes apresentaram algum grau de desnutrição ${ }^{15}$.

Estudos descrevem que o tempo de execução do TUG teste influencia diretamente na evolução clínica dos pacientes, visto que a redução de 1 segundo da velocidade equivale a $8 \%$ do aumento do risco de mortalidade.

O TUG teste executado pelos participantes foi de $11,8 \pm 0,70$ segundos, o que é considerado um valor abaixo do predito para potencial risco de queda, sendo o ponto de corte estabelecido de 12,47 segundos ${ }^{16,17}$. Isso nos sugere que a amostra demonstrou valores de segurança em relação ao teste, confirmado por $81,35 \%(n=48)$ da amostra que relata não ter sofrido nenhum tipo de queda nos últimos 12 meses; possivelmente pelo pouco tempo de tratamento em que esses pacientes foram submetidos, ainda não demonstrando avanços progressivos mais severos relacionados a incapacidade de locomoção.

Os resultados obtidos pelo WHODAS identificaram que os participantes apresentam grau de dificuldade leve para os domínios de cognição, relações interpessoais e atividades no trabalho; assim como dificuldade moderada para mobilidade, autocuidado e participação; além de dificuldade grave para atividades domésticas. A pontuação total do WHODAS demonstrou dificuldade moderado no score final.

Castro et al. ${ }^{7}$ fizeram a validação do instrumento WHODAS 2.0 com contingente de 51 pacientes hemodialíticos e constataram uma média total de pontuação do instrumento de 15,66 e \% de incapacidade de 12,33, o que sugere uma dificuldade leve no total, enquanto a atual amostra resultou com valores o dobro acima da referida, com média de 32,36 e \% de incapacidade de 30,20, classificada com uma dificuldade média. Percebemos também que o domínio mais afetado dentre as opções foi o relacionado a atividades domésticas, com valor de 55,59, enquanto no estudo citado, o domínio mais pontuado foi o relacionado a mobilidade, com escore de 31,12.

O TUG test possui uma interdependência proporcional com os domínios de cognição, mobilidade, autocuidado e participação, o que sugere que quanto maior o tempo para realização do teste, maior seria o comprometimento dos respectivos domínios, sendo assim, o declínio cognitivo, do cuidado com a saúde e da capacidade de se deslocar podem 
agravar o risco de queda desses pacientes, comprometendo a funcionalidade.

Outro fator de destaque é a correlação inversamente proporcional entre a FPP e os domínios de mobilidade, autocuidado e atividades domésticas, indicando que quanto menor a força do indivíduo, maior será sua incapacidade para situações de vida diária, como atividades domésticas e o cuidado de si mesmo, aumentando sua dependência funcional.

O grau de dor verificado pela escala EVA teve representatividade junto aos domínios de cognição, mobilidade, autocuidado, relações interpessoais, participação e atividades domésticas, propondo que quanto maior o nível de dor que o paciente hemodialítico relata, maior será seu grau de incapacidade.

Estudos prévios demonstram que as alterações provenientes tanto da doença em si, como as provocadas pelo tratamento, causam restrições e prejuízos a qualidade de vida desses pacientes ${ }^{8}$, afetando-os fisicamente, funcionalmente, emocional e espiritualmente, explicando então os resultados encontrados através do questionário WHODAS.

O tamanho da amostra pode ser considerado uma limitação da pesquisa, espera-se que futuramente seja realizado um update para testar nova força de associação, dentre elas a relação entre o paciente transplantado renal e força global com exames laboratoriais.

\section{CONCLUSÃ̃o}

Diante da avaliação clínico funcional e categorização da saúde dos pacientes com DRC submetidos à hemodiálise, bem como suas principais comorbidades e incapacidades encontradas nesse estudo, verifica-se a necessidade de os serviços de diálise proporcionarem atendimento especializado com suporte e acesso a uma equipe interdisciplinar. Ademais, a Fisioterapia dispõe de vários recursos de avaliação e intervenção, como também possui grandes vantagens em prol de amenizar os prejuízos da funcionalidade geralmente encontrados nessa população.

Participação dos autores: Thatyane Cruvinel Silva Hungria ${ }^{1}$. Co-autor e revisor: Gustavo da Silva Azevedo². Coleta de dados: Amanda Rafaella Araújo de Campos ${ }^{3}$. Análise estatística: Amélia Cristina Stival Duarte ${ }^{4}$

Não há conflitos de interesses entre os autores do artigo. Financiamento próprio. Aprovado pelo Comitê de Ética em Pesquisa sob número de parecer n 2.398.218. Estudo realizado no Hospital Estadual Dr. Alberto Rassi- HGG, Goiânia, Goiás, Brasil.

\section{REFERÊNCIAS}

1. Barreto FC, Stinghen AEM, Oliveira RB, Franco ATB, Moreno $\mathrm{AN}$, Barreto DV, et al Em busca de uma melhor compreensão da doença renal crônica: uma atualização em toxinas urêmicas. J Bras Nefrol. 2014;36(2):221-35. https://doi.org/10.5935/01012800.20140033

2. Amaral TLM, Amaral CA, Vasconcellos MTL, Monteiro GTR. Prevalência e fatores associados à doença renal crônica em idosos. Rev Saúde Pública. 2019;53:44. https://doi.org/10.11606/ s1518-8787.2019053000727.

3. Santos LF, Prado BC, Castro FPS, Brito RF, Maciel SC, Avelar TC. Qualidade de vida em transplantados renais. Psico-USF. 2018;23(1),163-72. https://doi.org/10.1590/S003489102004000500018 .

4. Marinho AWGB, Penha AP, Silva MT, Galvão TF. Prevalência de doença renal crônica em adultos no Brasil: revisão sistemática da literatura. Cad Saúde Coletiva. 2017;25(3): 379-88. https://doi.org/10.1590/1414-462x201700030134

5. Jesus NM, Souza GF, Mendes-Rodrigues C, Almeida NOP, Rodrigues DDM, Cunha CM. Qualidade de vida de indivíduos com doença renal crônica em tratamento dialítico. J Bras Nefrol. 2019;41(3):364-74. https://doi.org/10.1590/21758239-jbn-2018-0152

6. Oliveira JF, Marinho CLA, Silva RS, Lira GG. Qualidade de vida de pacientes em diálise peritoneal e seu impacto na dimensão social. Esc Anna Nery. 2019;23(1):e20180265. DOI: 10.1590/2177-9465-EAN-2018-0265

7. Castro SS, Leite CF, Baldin JE, Accioly MF. Validação da versão brasileira do WHODAS 2.0 em pacientes em tratamento hemodialítico. Fisioter Mov. 2018,31:e003130. https://doi.org/ 10.1590/1980-5918.031.ao30.

8. Mota LS, Oliveira CMC, Pinheiro JF, Martho L, Santos LCO, Nóbrega DG, et al .Estudo comparativo entre transplantes renais com doador falecido critério expandido e critério padrão em um único centro no Brasil. J Bras Nefrol. 2016;38(3):334-43. http://dx.doi.org/10.5935/0101-2800.20160051

9. Amorim RG, Guedes GS, Vasconcelos SML, Santos JCF. Doença renal do diabetes: cross-linking entre hiperglicemia, 
desequilíbrio redox e inflamação. Arq Bras Cardiol, 2019;112(5):577-87. http://dx.doi.org/10.5935/abc.20190077

10. Andrade DM, Chellini PR, Leite JB, Corrêa JOA. Análise das concentrações de ureia e creatinina em soro e plasma com ácido etilenodiamino tetra-acético e citrato de sódio. RBAC. Rev Bras Analis Clin. 2017;49(3):268-74. DOI: $10.21877 / 2448-3877.201700562$

11. Abensur H. Deficiência de ferro na doença renal crônica. Rev Bras Hematol Hemoter, 2010;32( Supp1 2):95-8. http://dx.doi.org/ $10.1590 /$ S1516-84842010005000047

12. Oliveira ACF, Vieira DSR, Bündchen DC. Nível de atividade física e capacidade funcional de pacientes com doença renal crônica pré-dialítica e em hemodiálise. Fisioter Pesqui. 2018;25(3):323-9. http://dx.doi.org/10.1590/18092950/18003625032018

13. Sallinen J, Stenholm S, Rantanen T, Heliövaara M, Sainio P, Koskinen S. Hand- Grip strength cut-points to screen older persons at risk for mobility limitation. J Am Geriatr Soc. 2010;58(9):1721-6. doi: 10.1111/j.1532-5415.2010.03035.x
14. Souza VA, Oliveira D, Mansur HN, Fernandes NMS, Bastos MG. Sarcopenia na doença renal crônica. J Bras Nefrol. 2015;37(1):98-105. https://doi.org/10.5935/01012800.20150014

15. Bousquet-Silva K, Costa LG, Andrade JLM. Estado nutricional de portadores de doença renal crônica em hemodiálise no Sistema Único de Saúde. Ciênc Saúde Coletiva. 2019;24(3):1189-99. https://doi.org/10.1590/141381232018243.11192017

16. Martinez BP, Santos MR,Simões LP, Ramos IR, Oliveira CS, Forgiarini JLA et al. Segurança e reprodutibilidade do teste timde up and go em idosos hospitalizados.Rev Bras Med Esporte. 2016;22(5):408-11. https://doi.org/10.1590/1517869220162205145497

17. Lopes,LCC.Efeito de diferentes intensidades do treinamento de força intradialítico sobre a massa muscular e capacidade funcional e qualidade de vida de pacientes em hemodiálise: ensaio clínico randomizado. [dissertação]. Goiânia: Programa de Pós Graduação em Nutrição e Saúde (FANUT), Universidade Federal de Goiás; 2016. Disponível em: https://repositorio.bc.ufg.br/ tede/bitstream/tede/5756/5/Disserta \%c3\%a7\%c3\%a3o\%20-\%20 Lorena\%20Cristina\%20curado\%20Lopes\%20-\%202016.pdf 